Pacific Journal of Mathematics

ASYMPTOTIC BEHAVIOR OF SOLUTIONS OF RETARDED 


\title{
ASYMPTOTIC BEHAVIOR OF SOLUTIONS OF RETARDED DIFFERENTIAL DIFFERENCE EQUATIONS
}

\author{
J. C. LiLlo
}

The asymptotic behavior of the solutions of nonautonomous $n^{\text {th }}$ order linear retarded differential difference equations is studied in this paper. It is shown that if the coefficients satisfy certain restrictions, then for any real $K$ there exists a finite dimensional subspace $F(K)$ of the solution space having the following property. For any solution $x$ of the equation one has for all $t>0$ that $x(t)=x_{K}(t)+x_{r}(t)$ where $x_{k}$ belongs to $F(K)$ and $x_{r}(t)=0(\exp (-K t))$ as $t \rightarrow \infty$. As in the author's earlier papers, considering the periodic and almost periodic cases, the spaces $F^{\prime}(K)$ are obtained by treating the nonautonomous equation as a perturbation of an $n^{\text {th }}$ order autonomous equation.

1. Introduction and notation. We consider perturbations of the autonomous $n^{\text {th }}$ order equation

$$
L_{0}(x(t))=x^{(n)}(t)+\sum_{k=0}^{m} \sum_{l=0}^{l(k)} c_{l k} x^{(l)}\left(t-\Delta_{k}\right)=0
$$

where $0=\Delta_{0}<\Delta_{1}<\Delta_{m}$ and the $c_{l k}$, for all pairs $(l, k)$ occurring in (1.1), are real numbers. We assume $m \geqq 1$. We also assume that $l(0)<n$ and that $l(k)<n, c_{l(k) k} \neq 0$ for $k=1, \cdots, m$. The perturbed equations will be of the form

$$
L_{0}(x(t))=D(x(t))
$$

where

$$
D(x(t))=\sum_{h=0}^{V} \sum_{g=0}^{g(h)} q_{g h}(t) x^{(g)}\left(t-\sigma_{h}\right) .
$$

Here it is assumed that the $q_{g h}$ belong to $C^{2 n}(-\infty, \infty)$ and there exists an $M_{1}>0$ such that

$$
\left|q_{g h}^{(j)}(t)\right| \leqq M_{1}
$$

for $t \in(-\infty, \infty), j \leqq 2 n$, and $(g, h) \in B$, where $B$ denotes the set of all prirs $(g, h)$ in (1.3) for which $q_{g h}(t) \not \equiv 0$.

In earlier papers, the author has established, in the cases where the coefficients $q_{g h}$ are periodic [6] or almost periodic [7], that for $K>0$ and sufficiently large there exist finite dimensional solution spaces $F(K)$ of (1.2) possessing the following properties. Any so- 
lution of (1.2) has a representation of the form

$$
x(t)=x_{K}(t)+x_{r}(t) .
$$

Here $x_{K} \in F(K)$ and there exists an $M(x)$ such that

$$
\left|x_{r}(t)\right|<M(x) \exp (-K t) \quad \text { for all } t \geqq 0 .
$$

If $H(K)$ denotes the corresponding subspace for (1.1) then there exists a sequence $a_{r}, \lim a_{r}=-\infty$ such that

$$
n\left(a_{r}\right)=\operatorname{dim} H\left(-a_{r}\right)=\operatorname{dim} F\left(-a_{r}\right) .
$$

In $\S 3$ we shall extend these results to systems of the form (1.2) under certain additional restrictions on the perturbations (1.3). In order to state these restrictions we next introduce the notion of a distribution diagram for (1.1) and (1.3).

Associated with (1.1) we have the characteristic polynomials

$$
P(z)=\sum_{k l} c_{l k} z^{l} \exp \left(-\Delta_{k} z\right)+z^{n}
$$

and $G(z)=P(z) \exp \left(\Delta_{m} z\right)$ where $\sum \sum_{k l}$ denotes the double sum occurring in (1.1). For $G(z)$ we define its distribution diagram [6] as follows. Let $S(G)$ denote the set of points, in a Cartesian plane, consisting of the point $\left(\Delta_{m}, n\right)$ and the points $\left(\Delta_{m}-\Delta_{k}, l\right)$ where $(l, k)$ is any pair of subscripts in (1.1) for which $c_{l k} \neq 0$. We denote by $p_{j}=\left(\beta_{j}, m_{j}\right)$, $j=0, \cdots, k$, those points in $S(G)$ which possess the following properties:

(a) $p_{0}=(0, l(m)), p_{k}=\left(\Delta_{m}, n\right)$

(b) $\beta_{j}<\beta_{j+1}$ and $m_{j}<m_{j+1}$ for $j=0,1, \cdots, k-1$

(c) if $l_{j}$ denotes the line segment connecting $p_{j-1}$ and $p_{j}$ then every point of $S(G)$ lies on or below at least one of the $l_{j}, j=1$, $\cdots, k$,

(d) if $u_{j}$ denotes the slope of $l_{j}$ then $u_{1}>u_{2}, \cdots,>u_{k}$.

The graph consisting of the line segments $l_{1}, \cdots, l_{k}$ is referred to as the distribution diagram of $G$. For $j=1, \cdots, k$ we denote by $G_{j}(z)$ those terms in $G(z)$ which correspond to points in $S(G)$ on the line segment $l_{j}$. Each of the polynomials $G_{j}(z)$ may be factored

$$
G_{j}(z)=b_{j} z^{m_{j-1}} \exp \left(\beta_{j-1} z\right) \prod_{h=1}^{\alpha(j)}\left(z \exp \left(z / u_{j}\right)-\gamma_{j h}\right)^{\beta(j, h)}
$$

where $\gamma_{j_{h}} \neq \gamma_{j i}$ for $i \neq h$ and $b_{j}$ denotes the coefficient in $G(z)$ of the term $z^{m_{j}} \exp \left(\beta_{j} z\right)$. Since our estimates for $|G(z)|$ depend directly on the numbers $\beta(j, h)$ in (1.9) we associate with $P(z)$ the following constant 


$$
\sigma(P)=\max \beta(j, h) \text { for } j=1, \cdots, k \text { and } h \in[1, \cdots, \alpha(j)]
$$

Definition. Equation (1.1) is said to satisfy condition $I \alpha$ if $\sigma(P)=\alpha$ for its associated polynomial $P(z)$ as defined in (2.1).

As mentioned earlier it is necessary to impose a restriction, condition $I I \alpha$, on the perturbation term (1.3). In order to define this condition we associate with (1.3) a distribution diagram. Let

$$
F(z)=\exp \left(\Delta_{m} z\right) \sum_{h g} \sum r_{g h} z^{g} \exp \left(-\sigma_{h} z\right)
$$

where $r_{g h}=1$ if $(g, h) \in B, r_{g h}=0$ if $(g, h) \notin B$, and $\sum_{g h}$ denotes the double sum in (1.3). As in the case of $G(z)$ one now defines the set of points $S(G)$ and then the distribution diagram of $F$ which is also referred to as the distribution diagram associated with (1.3).

Let $R(\sigma)$ denote the closed region bounded by the lines $x=\sigma / u_{k}$, $y=0, x=\Delta_{m}$, and the line segments $l_{1}(\sigma), \cdots, l_{k}(\sigma)$. Here $l_{j}(\sigma)$ is the line segment joining the points $p_{j}(\sigma)=\left(\beta_{j}+\sigma / u_{k}, m_{j}\right)$ and $p_{j-1}(\sigma)=\left(\beta_{j-1}+\sigma / u_{k}, m_{j-1}\right)$ where $p_{j}$ and $p_{j-1}$ are the endpoints of $l_{j}$ in the distribution diagram of $G(z)$.

Definition. We say that (1.3) satisfies conditions $I I_{\alpha}$ for any real $\alpha$ if its distribution diagram is contained in $R(\sigma)$ for some $\sigma>\alpha$.

For an intuitive discussion of condition $I I_{\alpha}$ the reader is referred to the author's earlier paper [6]. Before stating the estimates that we shall need in $\S 2$ we introduce the following notation.

For any complex number $z_{1}$ we have

$$
\begin{aligned}
& k\left(z_{1}\right)=\left|\exp \left(-z_{1} / u_{k}\right)\right| \text { and } \\
& l\left(z_{1}\right)=\left\{z: \operatorname{Re}(z)=\operatorname{Re}\left(z_{1}\right)\right\} .
\end{aligned}
$$

For any pair $(g, h) \in B$ we define

$$
H(g, h, w, z)=\exp \left[-\Delta_{h}(z-i w)\right](z-i w)^{j} / P(z-i w) .
$$

Let $Z$ denote the set of zeros of $P(z)$. In an earlier work [6] the author has shown that there exists a decreasing sequence or real numbers $\left\{a_{r}\right\}, \lim a_{r}=-\infty, a_{0}-2$ lies to the right of $Z$ and 1 , and an $M_{4}$ such that the strips $S_{r}=\left\{z:\left|\operatorname{Re} z-a_{r}\right|<M_{4} k^{-1}\left(a_{r}\right)\right\}$ do not contain any points of $Z$. Using the estimates established in [6] one has the following results. If (1.1) satisfies $I_{\alpha}$ and (1.3) satisfies $I I_{\alpha+\gamma}$, $\gamma \geqq 0$, then there exists $\beta>0, H_{1}>0$ such that for any pair $(g, h) \in B, r \geqq H_{1}$ and $\delta_{r}=M_{4} k^{-1}\left(a_{r}\right) / 4$ one has the estimates

$$
\mid H(g, h, 0, z) \leqq k^{-(\beta+r)}\left(a_{r}\right) \text { for } \operatorname{Re} z \in\left(a_{r}-\delta_{r}, a_{r}+\delta_{r}\right)=\alpha_{r}
$$




$$
\begin{aligned}
& \int|H(g, h, 0, z)|^{2}|d z| \leqq k^{-2(\beta+\gamma)+1}\left(a_{r}\right) \\
& \int|H(g, h, 0, z)||d z| \leqq k^{-(\beta+\gamma)+1}\left(a_{r}\right)
\end{aligned}
$$

where the integrals in (1.16) and (1.17) are along any line $l\left(z_{1}\right)$ for $\operatorname{Re} z_{1} \in \alpha_{r}$. We also have that there exists an $M \geqq 0$ such that for any $\sigma \geqq 0$

$$
\begin{gathered}
|H(g, h, 0, z)| \leqq M /|z|^{2} \quad \text { for } z \in l\left(a_{0}+\sigma\right) \\
\int|H(g, h, 0, z)||d z| \leqq M /\left(a_{0}+\sigma\right) \\
\int|H(g, h, 0, z)|^{2}|d z| \leqq M /\left(a_{0}+\sigma\right)^{3}
\end{gathered}
$$

where the integrals in (1.19) and (1.20) are along the line $l\left(a_{0}+\sigma\right)$.

2. The Greens function. In this section we shall obtain in Theorem 2.1 a representation result for the Greens function associated with equation (1.2). The proof of Theorem 2.1 consists of treating equation (1.2) as a perturbation of (1.1) and solving by successive approximations. To facilitate this discussion we introduce the following notation. Let $G(t, s)$, called the Greens function, denote the function which vanishes for $t<s$, satisfies (1.2) as a function of $t$ for $t>s, G^{(n-1)}\left(\underline{s}^{+}, s\right)-G^{(n-1)}\left(\underline{s}^{-}, s\right)=1$ and $G^{(j)}(\underline{t}, s)$ is continuous in $t$ for all $j \leqq n-2$. Here $f^{(j)}(\underline{t}, s)$ denotes the $j^{\text {th }}$ partial derivative of $f(t, s)$ with respect to $t$. The $j^{\text {th }}$ partial derivative of $f(t, s)$ with respect to $s$ will be denoted by $f^{(j)}(t, s)$. We shall denote by $G(0, t, s)$ the Greens function for equation (1.1). Associated with $G(0, t, s)$ we have

$$
\begin{array}{r}
G(0, t, s, r, 1)=\int_{r} \exp [z(t-s)] \Delta(z) d z \text { for all values of } t, \\
G(0, t, s, r, 2)=\int_{a_{r}} \exp [z(t-s)] \Delta(z) d z \quad \text { for } t>s,
\end{array}
$$

where $\Delta(z)=1 / P(z)$ and the integrals are defined as follows. The symbol $\int_{a_{r}}$ denotes the line integral $1 /(2 \pi i) \int_{a_{r}-\infty i}^{a_{r+i}}$ and $\int_{a_{\bar{r}}}$ denotes the negative of this integral. The symbol $\int_{r}^{a_{r}}$ denotes the sum of the two integrals $\int_{a_{\bar{r}}}$ and $\int_{a_{0}}$ where the $a_{j}$ are defined as in $\S 1$. Since $Z$ contains only a finite set of points to the right of $l\left(a_{r}\right)$ it follows easily [1] that

$$
G(0, t, s)=G(0, t, s, r, 1)+G(0, t, s, r, 2)
$$


for all values of $t$. Here $G(0, t, s, r, 1)$ satisfies equation (1.1) for all values of $t$ and $G(0, t, s, r, 2)$ satisfies equation (1.1) for all $t>s$. It also follows [1] that for any $k \geqq 0$ and $r$ sufficiently large that there exist constants $M(k, r)$ such that for $j \leqq n-1$

$$
\begin{aligned}
&\left|G^{(j)}(0, \underline{t}, s, r, 1)\right| \leqq M(k, r) \exp [b(t-s)] \\
& \text { for } t \geqq s \text { and } b \in[a+k, a+k+1] \\
&\left|G^{(j)}(0, \underline{t}, s, r, 1)\right| \leqq M(k, r) \exp [\gamma(t-s)] \quad \text { for } t \leqq s \text { and } \gamma \in \alpha_{r} \\
&\left|G^{(j)}(0, \underline{t}, s, r, 2)\right| \leqq M(k, r) \exp [\alpha(t-s)] \\
& \text { for } t \geqq s \text { and } \alpha \in \alpha_{r} .
\end{aligned}
$$

Since $G(0, t, s, r, 1)$ and $G(0,, t, s, r, 2)$ satisfy equation (1.1) for $t$ and $s$ in appropriate domains it follows that except for a finite set of points in $\left[0,(n+1) \Delta_{m}\right]$ the derivatives of order $j \leqq 2 n$ will exist and the constants $M(k, r)$ may be chosen so that one has for these higher derivatives the estimates (2.4) for the indicated values of $t$ and $s$.

We now establish, in Theorem 2.1, a similar representation result for $G(t, 0)$. For $i=1,2$ we define $G(0, t, r, i) \equiv G(0, t, 0, r, i)$. For $j \geqq 1$ we define for all values of $t$

$$
\begin{aligned}
G(j, t, r, 1)= & \int_{-\infty}^{t} D(G(j-1, w, r, 1)) G(0, t-w, r, 2) d w \\
& +\int_{0}^{t} D(G(j-1, w, r, 1)) G(0, t-w, r, 1) d w \\
& +\int_{0}^{\infty} D(G(j-1, w, r, 2)) G(0, t-w, r, 1) d w
\end{aligned}
$$

and for $t>0$

$$
\begin{aligned}
G(j, t, r, 2)= & \int_{\infty}^{t} D(G(j-1, w, r, 2)) G(0, t-w, r, 1) d w \\
& +\int_{0}^{-\infty} D(G(j-1, w, r, 1)) G(0, t-w, r, 2) d w \\
& +\int_{0}^{t} D(G(j-1, w, r, 2)) G(0, t-w, r, 2) d w
\end{aligned}
$$

For $t \leqq 0, j \geqq 1$ we set $G(j, t, r, 2)=-G(j, t, r, 1)$. We then set

$$
\begin{aligned}
& G(t, r, 1)=\sum_{j=1}^{\infty} G(j, t, r, 1) \\
& G(t, r, 2)=\sum_{j=0}^{\infty} G(j, t, r, 2) .
\end{aligned}
$$

THEOREM 2.1. If equation (1.1) satisfies $I_{\alpha}$ and Equation (1.3) 
satisfies $I I_{\alpha+2}$ then there exists an $H_{1}>0$ such that for any $r \geqq H_{1}$ the function $G(t, 0)$ can be written for all values of $t$

$$
G(t, 0)=G(t, r, 1)+G(t, r, 2) .
$$

The function $G(t, r, 1)$ satisfies equation (1.2) for all values of $t$ and $G(t, r, 2)$ satisfies (1.2) for all $t>0$. Furthermore there exists $a$ constant $k$ and constants $N(k, r)$ for $r \geqq H_{1}$ such that

$$
\begin{aligned}
&\left|G^{(j)}(t, r, 1)\right| \leqq N(k, r) \exp (b t) \\
& \quad \quad \text { for } t \geqq 0, b \in\left[a_{0}+k, a_{0}+k+1\right] \\
&\left|G^{(j)}(t, r, 1)\right| \leqq N(k, r) \exp (\gamma t) \\
& \quad \text { for } t \leqq 0, \gamma \in\left[a_{r}-\delta_{r} / 2, a_{r}+\delta_{r} / 2\right]=\beta_{r} \\
&\left|G^{(j)}(t, r, 2)\right| \leqq N(k, r) \exp (\alpha t) \quad \text { for } t \geqq 0, \alpha \in \beta_{r} .
\end{aligned}
$$

Proof. For any function $f$ we denote by $[f]^{+}$the function which equals $f$ for $t \geqq 0$ and is zero for $t<0$. We denote by $[f]^{-}$the function $f-[f]^{+}$. We denote by $\|f\|_{1}$ and $\|f\|_{2}$ the $L^{1}(-\infty, \infty)$ $L^{2}(-\infty, \infty)$ norms of $f$. We also introduce the functions

$$
f(\gamma, m, h, g, r, j, t) \equiv \exp (-\gamma t) G^{(g)}\left(m, t-\sigma_{h}, r, j\right) .
$$

Then using (2.4), (2.9), and (1.17) it follows for $\gamma \in \beta_{r}, r$ sufficiently large, and any pair $(g, h) \in B$

$$
\begin{aligned}
& \left\|[f(\gamma, 0, h, g, r, 1,-)]^{-}\right\|_{1}<k^{-\beta / 2}\left(a_{r}\right) \\
& \left\|[f(\gamma, 0, h, g, r, 2,-)]^{+}\right\|_{1}<k^{-\beta / 2}\left(a_{r}\right)
\end{aligned}
$$

and the $L^{2}[-\infty, \infty]$ norms of the above functions are bounded by $k^{-\beta / 2-3 / 2}\left(a_{r}\right)$. Similarly using (1.19) in place of (1.17) for any $k>0$, $(g, h) \in B$ and $b \in\left[a_{0}+k, a_{0}+k+1\right]$ it follows that

$$
\left\|[f(b, 0, h, g, 1,-)]^{+}\right\|_{1}<M /\left[a_{0}+k\right]
$$

and its $: L^{2}[-\infty, \infty]$ norm is bounded by $M^{1 / 2} /\left(a_{0}+k\right)^{3 / 2}$. Next we note that if $f \in L^{1}[-\infty, \infty], g \in L^{2}[-\infty, \infty]$ then

$$
h(y)=\int_{-\infty}^{\infty} f(y+x) g(x) d x
$$

belongs to $L^{2}[-\infty, \infty]$ and

$$
\begin{aligned}
& |h(y)| \leqq\|f\|_{2}\|g\|_{2} \\
& \|h\|_{2} \leqq\|f\|_{1}\|g\|_{2} .
\end{aligned}
$$

For $k=1,2$ let $C(k, m, \gamma, b)$ denote $\max \left(\left\|[f(\gamma, m, h, g, r, 2, \cdot)]^{+}\right\|_{k}\right.$, $\left\|[f(\gamma, m, h, g, r, 1, \cdot)]^{-}\right\|_{k},\left\|[f(b, m, h, g, r, 1, \cdot)]^{+}\right\|_{k}$ where the max is over all pairs $(g, h) \in B$, and $\gamma \in \beta_{r}$. 
Then from (2.5), (2.6), (2.10), (2.13) we have for all $(g, h) \in B$, $m \geqq 1$ that

$$
\begin{gathered}
\left\|[f(b, m, h, g, r, 1, \cdot)]^{+}\right\|_{2} \leqq 2 M_{1} v(n-3) C(2, m-1, \gamma, b) \\
{\left[k^{-\beta / 2}\left(a_{r}\right)+M /\left[a_{0}+k\right]\right]} \\
\left\|[f(\gamma, m, h, g, r, 1, \cdot)]^{-}\right\|_{2} \\
\leqq 3 M_{1} v(n-3) C(2, m-1, r, b) k^{-\beta / 2}\left(a_{r}\right) \\
\left\|[f(\gamma, m, h, g, r, 2, \cdot)]^{+}\right\|_{2} \\
\leqq 3 M_{1} v(n-3) C(2, m-1, \gamma, b) k^{-\beta / 2}\left(a_{r}\right) .
\end{gathered}
$$

Thus for $m \geqq 1$ one has

$$
\begin{aligned}
C(2, m, \gamma, b) \leqq & M_{1} v(n-3)\left[3 k^{-\beta / 2}\left(a_{r}\right)\right. \\
& \left.+2 M /\left[a_{0}+k\right]\right] C(2, m-1, \gamma, b) .
\end{aligned}
$$

Similarly from (2.12) we obtain the estimates for all values of $t$

$$
\begin{aligned}
\mid f(b, m, h, g, r, 1, t)]^{+} \mid \leqq & M_{1} v(n-3) C(2, m-1, \gamma, b) \\
& \times\left[3 k^{-\beta / 2-3 / 2}\left(a_{r}\right)+2 M^{1 / 2} /\left[a_{0}+b\right]^{3 / 2}\right] \\
\left|[f(\gamma, m, h, g, r, 1, t)]^{-}\right| & \\
\leqq & 3 M_{1} v(n-3) C(2, m-1, \gamma, b) k^{-\beta / 2-3 / 2}\left(a_{r}\right) \\
\left|[f(\gamma, m, h, g, r, 2, t)]^{+}\right| & \\
\leqq & 3 M_{1} v(n-3) C(2, n-1, \gamma, b) k^{-\beta / 2-3 / 2}\left(a_{r}\right) .
\end{aligned}
$$

Thus it follows that if $k$ and $r$ are chosen so that

$$
M_{1} v(n-3)\left[3 k^{-\beta / 2}\left(a_{r}\right)+2 M /\left[a_{0}+k\right] \mid<\frac{1}{2}\right.
$$

then the series in (3.7) will converge uniformly and absolutely in every finite interval and for $j=0$ and $\gamma=\alpha$ one has the estimates (2.9). For $m=0, j=1,2$ and $r>H_{1}$ we note that the functions $G(m, t, r, j)$ are independent of the choice of $\gamma \in \beta_{r}$. By induction this then holds for $m \geqq 1$. Thus we have the estimates in (2.9) for the case $j=0$. It also follows that $G(t, r, 2)$ and $G(t, r, 1)$ satisfy the equations

$$
\begin{aligned}
G(t, r, 1)= & \int_{-\infty}^{t} D(G(w, r, 1)) G(0, t-w, r, 2) d w \\
& +\int_{0}^{t} D(G(w, r, 1)) G(0, t-w, r, 1) d w+\int_{0}^{\infty} D(G(w, r, 2)) \\
& \times G(0, t-w, r, 1) d w \text { for all values of } t
\end{aligned}
$$

(2.18) $G(t, r, 2)=\int_{\infty}^{t} D(G(w, r, 2)) G(0, t-w, r, 2) d w$

$$
\begin{aligned}
& +\int_{0}^{-\infty} D(G(w, r, 1)) G(0, t-w, r, 2) d w \\
& +\int_{0}^{\infty} D(G(w, r, 2)) G(0, t-w, r, 1) d w \text { for } t \geqq 0 .
\end{aligned}
$$


Now using the estimates (2.4) it follows from (2.18) that $G(t, r, 1)$ satisfies equation (1.2) for all values of $t$ and $G(t, r, 2)$ satisfies equation (1.2) for $t>0$. It also follows from (2.18), the estimates for the terms $D(G(w, r, i))$ above, and (2.17) that the estimates given in (2.9) hold for $j \leqq n-1$ with $N(k, r)=2 M(k, r)$. This completes the proof of Theorem 2.1.

Associated with equations (1.1) and (1.2) we have the adjoint equations (2.19) and (2.20) given below. These equations are adjoint relative to the inner product given by (3.1) in the next section.

$$
\begin{gathered}
L_{0}^{+}(y(s))=y^{(n)}(s)+\sum_{k l} \sum(-1)^{l+n} c_{l k} y^{(l)}\left(s+\Delta_{k}\right) \\
L_{0}^{+}(y(s))=D^{+}(y(s))=\sum_{h g} \sum(-1)^{g+n}\left[q_{g h}\left(s+\sigma_{h}\right) y\left(s+\sigma_{h}\right)\right]^{(g)}
\end{gathered}
$$

Now in place of the equations (3.5) and (3.6) we have for $j \geqq 1$ and all value of $t$

$$
\begin{aligned}
G(j, t, s, r, 1)= & \int_{-\infty}^{t} D(G(j-1, w, s, r, 1)) G(0, t, w, r, 2) d w \\
& +\int_{s}^{t} D(G(j-1, w, s, r, 1)) G(0, t, w, r, 1) d w \\
& +\int_{s}^{\infty} D(G(j-1, w, s, r, 2)) G(0, t, w, r, 1) d w
\end{aligned}
$$

and for $t \geqq s$

$$
\begin{aligned}
G(j, t, s, r, 2)= & \int_{\infty}^{t} D(G(j-1, w, s, r, 2)) G(0, t, w, r, 1) d w \\
& +\int_{s}^{-\infty} D(G(j-1, w, s, r, 1)) G(0, t, w, r, 2) d w \\
& +\int_{s}^{t} D(G(j-1, w, s, r, 2)) G(0, t, w, r, 2) d w .
\end{aligned}
$$

For $t<s$ and $j \geqq 1$ we set $G(j, t, s, r, 2)=-G(j, t, s, r, 1)$. Then defining

$$
\begin{aligned}
& G(t, s, r, 1)=\sum_{j=0}^{\infty} G(j, t, s, r, 1) \\
& G(t, s, r, 2)=\sum_{j=0}^{\infty}(G(j, t, s, r, 2)
\end{aligned}
$$

we have the following theorem.

THEOREM 2.2. If equation (1.1) satisfies $I_{\alpha}$ and equation (1.3) satisfies $I I_{\alpha+2}$ for some $\alpha$ then there exists $H_{2}>0$ such that for any $r \geqq H_{2}$ the function $G(t, s)$ can be written for all value of $t, s$ in the form 


$$
G(t, s)=G(t, s, r, 1)+G(t, s, r, 2) .
$$

The function $G(t, s, r, 1)$ satisfies equation (1.2) as a function of $t$ and equation (2.20) as a function of $s$ for all values of $s$ and $t$. The function $G(t, s, r, 2)$ satisfies (1.2) as a function of $t$ and (2.20) as a function of $s$ for all $t>s$. The mixed partials $\partial^{i+j} G(t, s, r, 1) / \partial t^{i} \partial s^{j}$ are continuous and independent of order for all $i \leqq n-1, j \leqq n-1$, and all values of t and s. Furthermore these partials satisfy estimates of the form (2.9) with appropriate constants $N^{*}(k, r)$ and $t$ replaced by $t-s$ on the right side of the inequalities.

Proof. We first note that the change of variable $t_{1}=t-s$ converts the equations (2.21), (2.22) into equations of the form (2.18) where the coefficients used in defining $D$ have been shifted. Since the estimates on the coefficients were uniform in $t$ we have from Theorem 2.1 the assertions that $G(t, s, r, 1)$ and $G(t, s, r, 2)$ are solutions as functions of $t$, of equation (1.2) and satisfy equations (2.25), (2.26) for the appropriate values of $t$.

$$
\begin{aligned}
G(t, s, r, 1)= & \int_{-\infty}^{t} D(G(w, s, r, 1)) G(0, t, w, r, 2) d w \\
& +\int_{s}^{t} D(G(w, s, r, 1)) G(0, t, w, r, 1) d w \\
& +\int_{s}^{\infty} D(G(w, s, r, 2)) G(0, t, w, r, 1) d w \\
G(t, s, r, 2)= & \int_{\infty}^{t} D(G(w, s, r, 2)) G(0, t, w, r, 1) d w \\
& +\int_{s}^{-\infty} D(G(w, s, r, 1)) G(0, t, w, r, 2) d w \\
& +\int_{s}^{t} D(G(w, s, r, 2)) G(0, t, w, r, 2) d w .
\end{aligned}
$$

The results of Theorem 2.1 also justify integrating the equations (2.21) and (2.22) by parts, obtaining

$$
\begin{aligned}
& G(j, t, s, r, 1) \\
& =\int_{-\infty}^{t} \sum_{g h} G\left(j-1, w-\sigma_{h}, s, r, 1\right)\left[q_{g h}(w) G(0, t, w, r, 2)\right]^{(g)} d w \\
& \quad+\int_{s}^{t} \sum_{g h} G\left(j-1, w-\sigma_{h}, s, r, 1\right)\left[q_{g h}(w) G(0, t, w, r, 1)\right]^{(g)} d w \\
& \quad+\int_{s}^{\infty} \sum_{g h} G\left(j-1, w-\sigma_{h}, s, r, 2\right)\left[q_{g h}(w) G(0, t, w, r, 1)\right]^{(g)} d w
\end{aligned}
$$

and 


$$
\begin{aligned}
& G(j, t, s, r, 2) \\
& =\int_{\infty}^{t} \sum_{g h} G\left(j-1, w-\sigma_{h}, s, r, 2\right)\left[q_{g h}(w) G(0, t, w, r, 1)\right]^{(g)} d w \\
& \quad+\int_{s}^{-\infty} \sum_{g h} G\left(j-1, w-\sigma_{h}, s, r, 1\right)\left[q_{g h}(w) G(0, t, w, r, 2)\right]^{(g)} d w \\
& \quad+\int_{s}^{t} \sum_{g h} G\left(j-1, w-\sigma_{h}, s, r, 2\right)\left[q_{g h}(w) G(0, t, w, r, 2)\right]^{(g)} d w
\end{aligned}
$$

where []$^{(g)}$ denotes the $g^{\text {th }}$ derivative with respect to $w$. But then it follows from (2.27) and (2.28) for $i=1$ that

$$
L_{0}^{+} G(i, t, s, r, 1,)=D^{+}(G(i-1, t, s, r, 1))
$$

for all values of $t$ and $s$, and for $t \geqq s$ that

$$
L_{0}^{+} G(i, t, s, r, 2)=D^{+}(G(i-1, t, s, r, 2)) .
$$

Then assuming the relations (2.29) and (2.30) hold for $i=n$ one extends them to $i=n+1$. This is done by noting that due to the smoothness properties of $G(n, t, s, r, k), k=1,2$, and its derivatives for $n \geqq 1$ and the identity $G(n, t, s, r, 2)=-G(n, t, s, r, 1)$ for $t<s$ one may commute the operator $L_{0}^{+}$with the integral and summation signs in equations (2.27) and (2.28). Then by the induction hypothesis one obtains $D^{+} G\left(n-2, w-\sigma_{h}, s, r, k\right)$ inside the integral as $k=1$ or 2 in the given integral. But then, for the reasons mentioned above, one is able to commute $D^{+}$with the integral and summation signs to obtain $D^{+} G\left(n-1, w-\sigma_{h}, s, r, 1\right)$ in $(2.27)$ and $D^{+} G(n-1$, $\left.w-\sigma_{h}, s, r, 2\right)$ in (2.28). This completes the desired induction. The uniform convergence results of Theorem 2.1 now establish the assertions that $G(t, s, r, 1)$ and $G(t, s, r, 2)$ satisfy equation (2.20) for $t$ and $s$ in the appropriate domains. The assertions concerning the mixed partial derivatives of $G(t, s, r, 1)$ are obtained from the identity

$$
\begin{aligned}
& G(t, s, r, 1) \\
& =\int_{-\infty}^{t} \sum_{g h} G\left(w-\sigma_{h}, s, r, 1\right)\left[q_{g h}(w) G(0, t, w, r, 2)\right]^{(g)} d w \\
& \quad+\int_{s}^{t} \sum_{g h} G\left(w-\sigma_{h}, s, r, 1\right)\left[q_{g h}(w) G(0, t, w, r, 1)\right]^{(g)} d w \\
& \quad+\int_{s}^{\infty} \sum_{g h} G\left(w-\sigma_{h}, s, r, 2\right)\left(q_{g h}(w) G(t, w, r, 1)\right]^{(g)} d w .
\end{aligned}
$$

In light of the existence of the partials of $G(t, s, r, k)$ and $G(0, t, s, r, k)$ with respect to $s$ and $t$ and the fact that they satisfy bounds of the form (2.4), (2.9), the mixed partials $\partial^{i+j} / \partial t^{i} \partial s^{j}$ are obtained by taking these partials inside the integrals along with the additional terms due to the simple discontinuities of $G^{(n-1+k)}(0, \underline{t}, w, r, 2)$ at the finite set 
of isolated points $t_{k j}$ in the interval $\left[0,(n+1) \Delta_{m}\right]$. These terms are of the form $G^{(l)}\left(t_{k j}-\sigma_{h}, \underline{s}, r, 1\right)$ multiplied by appropriate constants where $l=g+i+j-(n-1+k)$. Thus the mixed partials possess the stated properties. This completes the proof of Theorem 2.2.

We now denote by $K$ the $\max g(h)$ for $h=0, \cdots, v$. We then have the following result.

THEOREM 2.3. The conclusions of Theorem 2.2 remain valid if the hypothesis that (1.3) satisfies $I I_{\alpha+2}$ is replaced by the hypothesis that (1.3) satisfies $I I_{\alpha+1 / 2}$ and the assumption that for all $j \leqq k$ and pairs $(g, h) \in B$ one has that $q_{g h}^{(j)}$ are in $L^{1}[-\infty, \infty]$.

Proof. Referring to the proof of Theorem 2.1 we note that in obtaining the estimates (2.14) we used (2.13). The restriction $I I_{\alpha+2}$ was used in obtaining a bound for the $L^{1}$ norm of the terms playing the role of $f$. But now using the fact that the $q_{g h} \in L^{1}[-\infty, \infty]$ one can obtain for the terms playing the role of $g$, in Theorem 2.1, an estimate on their $L^{1}$ norm. Thus one needs only an estimate on the $L^{2}$ norm of the terms playing the role of $f$ in the proof of Theorem 2.1. But for this it is sufficient to have (1.3) satisfy the condition $I I_{\alpha+1 / 2}$. Thus the results of Theorem 2.1 remain valid if the $q_{g h} \in$ $L^{1}[-\infty, \infty]$ for all pairs $(g, h) \in B$. The additional hypothesis that the $q_{g h}^{(j)} \in L^{1}[-\infty, \infty]$ for $j \leqq k$ are needed in the proof of Theorem 2.2 where the adjoint equation is considered. In particular, they are required for the representation given by equation (2.30). This completes the proof of Theorem 2.3.

3. Representation result. In this section we shall establish the representation result given by equation (1.5) and described in $\$ 1$. This is done by showing that the function $G(t, s, r, 1)$, occurring in Theorem 2.2 defines the finite dimensional subspace $F\left(-a_{r}\right)$ described in $\S 1$. This result is then used to establish Theorem 3.2.

Let $C$ denote the space of functions having $n-1$ continuous derivatives on $\left[-\Delta_{m}, 0\right]$ with the uniform norm $\|\cdot\|$ and $C^{+}$denote the space of functions having $n-1$ continuous derivatives on $\left[0, \Delta_{m}\right]$ along with the uniform norm. Then associated with equation (1.2) and its adjoint equation (2.20) we have for every real number $\sigma$ and $f \in C, d \in C^{+}$the following inner product

$$
\begin{aligned}
{[f, d, \sigma]=} & \sum_{i=1}^{n}(-1)^{i-1} f^{(n-i)}(0) d^{(i-1)}(0) \\
& -\sum_{k l}\left\{\int_{-\Delta_{k}}^{0} f^{(l)}(\xi) c_{l k} d\left(\xi+\Delta_{k}\right)\right.
\end{aligned}
$$




$$
\begin{aligned}
& \left.+\sum_{i=1}^{l}(-1)^{i} f^{(l-i)}(0) c_{l k} d^{(i-1)}\left(\Delta_{k}\right)\right\} \\
& +\sum_{h g}\left\{\int_{-\sigma_{h}}^{0} f^{(g)}(\xi) q_{g h}\left(\sigma+\xi+\sigma_{h}\right) d\left(\xi+\sigma_{h}\right) d \xi\right. \\
& \left.\left.+\sum_{i=1}^{g}(-1)^{i} f^{(l-i)}(0)\left[q_{g h}\left(\sigma+\sigma_{h}\right) d\left(\sigma_{h}\right)\right]^{(i-1)}\right]\right\} .
\end{aligned}
$$

Here we have written

$$
\left[q_{g h}\left(\sigma+\sigma_{h}\right) d\left(\sigma_{h}\right)\right]^{(j)} \quad \text { for } \quad \frac{d^{j}\left[q_{g h}\left(\sigma+\sigma_{h}+\xi\right) d\left(\xi+\sigma_{h}\right)\right]}{d \xi^{j}}
$$

evaluated at $\xi=0$. If $y$ denotes a solution of the adjoint equation (2.20) for $s \leqq s_{0}$, then for any $\sigma \leqq s_{0}$ we define $y_{\sigma} \in C^{+}$by the equation $y_{o}(\xi)=y(\sigma+\xi)$ for $\xi \in\left[0, \Delta_{m}\right]$. Similarly if $x$ is a solution of (1.2) for $t \geqq t_{0}$ we denote by $x_{\sigma}, \sigma \geqq t_{0}$, the function $x_{0} \in C$ defined by $x_{\sigma}(\xi)=x(\sigma+\xi)$ for $\xi \in\left[-\Delta_{m}, 0\right]$. For any $r, r \geqq H_{2}$ as given in Theorem 2.2, we define the linear operator $E(r)$ on $C$ by setting

$$
E(r, f)(t)=\left[f, G_{0}(t, \cdot, r, 1), 0\right] \text { for } t \in\left[-\Delta_{m}, 0\right] .
$$

We then have the following result.

THEOREM 3.1. For any $r \geqq H_{2}$ the linear operator $E(r)$ defines a projection of $C$ into $C$. The range of $E(r)$ has the dimension $n\left(a_{r}\right)$ as defined in $\S 1$.

Proof. We first establish the identity

$$
G(t, s, r, 1)=\left[G_{0}(\cdot, s, r, 1), G_{0}(t, \cdot, r, 1), 0\right]
$$

for $r \geqq H_{2}$ and all values of $t$ and $s$. Since the mixed partials of $G(t, s, r, 1)$ of order up to $2 n$ are continuous and since $G(t, s, r, 1)$ is a solution of (1.2) and (2.20) it follows that the right side of (3.3) is a solution of (1.2), as a function of $t$, and a solution of (2.20), as a function of $s$ for all values of $s$ and $t$. We consider (3.3) for the case in which $t>\Delta_{m}$. For these values of $s$ and $t$ [4] one has the identity

$$
\begin{aligned}
G(t, s, r, 1)= & {\left[G_{0}(\cdot, s, r, 1), G_{0}(t, \cdot), 0\right] } \\
= & {\left[G_{0}(\cdot, s, r, 1), G_{0}(t, \cdot r, 1), 0\right] } \\
& +\left[G_{0}(\cdot, s, r, 1), G_{0}(t, \cdot r, 2), 0\right] .
\end{aligned}
$$

Now it is known [4] that for all values of $\sigma \leqq 0$

$$
\left[G_{o}(\cdot, s, r, 1), G_{\sigma}(t, \cdot, r, 2), \sigma\right]
$$

is a constant which is independent of $\sigma$. From Theorem 2.2 it follows 
that there exists an $M>0$ such that (3.5) is bounded by

$$
M \exp \left[\left(a_{r}-\delta_{r / 2}\right)(t-\sigma)\right] \exp \left[\left(a_{r}+\delta_{r / 2}\right)(\sigma-s)\right] .
$$

Letting $\sigma \rightarrow-\infty$ we have that the expression in (3.5) is zero and so we obtain (3.3) for all $t>\Delta_{m}$. Using the fact that solutions of (1.2) have unique backward continuations [5], [4], the identity (3.3) follows for all values of $t$. Using the continuity of the mixed partials of $G(t, s, r, 1)$ as given in Theorem 2.2 and Fubini's theorem one may carry out the interchanges in the orders of integrations and differentiations [6] to obtain the identity

$$
\begin{aligned}
& {\left[\left[f, G_{0}(t, \cdot r, 1), 0\right], G_{0}(\alpha, \cdot r, 1), 0\right]} \\
& \quad=\left[f,\left[G_{0}(\cdot, s, r, 1), G_{0}(\alpha, \cdot r, 1), 0\right], 0\right]
\end{aligned}
$$

where $t$ varies in $\left[-\Delta_{m}, 0\right]$ and $s$ in $\left[0, \Delta_{m}\right]$. Thus we have that $E(r, E(r, f))=E(r, f)$ and it follows that $E(r)$ defines a projection on $C$. In order to prove that the dimension of the range of $E(r)$ is $n\left(a_{r}\right)$ one introduces the one parameter family of equations

$$
L_{0}(x(t))=u D(x(t)) \text { for } 0 \leqq u \leqq 1 .
$$

Then from Theorem 2.2 one again obtains a function $G(t, s, r, 1, u)$ which for $u=1$ reduces to $G(t, s, r, 1)$. As in [6], the inner product (3.1) is modified to $[f, d, \sigma, u]$ by replacing the functions $q_{g h}$ by the functions $u q_{g h}$. One again shows that

$$
E(r, f, u)(t)=\left[f, G_{0}(t, \cdot, r, 1, u), 0, u\right]
$$

defines a projection. It then follows from the proof of Theorems 2.1 and 2.2 that the mixed partials $G^{j}(t, s, r, 1, u)$ are continuous functions of $u$ for $|t| \leqq 2 \Delta_{m},|s| \leqq 2 \Delta_{m}$, and $j \leqq 2 n$. Thus it follows that the norm of $E(r, u)$ is a continuous function of $u$ for $0 \leqq u \leqq 1$. Since the dimension of the range of $E(r, 0)$ is $n\left(a_{r}\right)$ it then follows by the usual arguments [6] that the dimension of the range of $E(r, 1)$ is also $n\left(a_{r}\right)$. This completes the proof of Theorem 3.1.

Let $C(r)$ denote the range of $E(r)$. Then since $C(r)$ has the dimension $n\left(a_{r}\right)$ we may select a basis $\zeta_{r_{j}}, j=1, \cdots, n\left(a_{r}\right)$ for $C(r)$. Thus for every $f \in C(r)$ we have a unique representation of the form

$$
E(r, f)=\sum_{j=1}^{n\left(a_{r}\right)} a(f, j) \zeta_{r_{j}}
$$

Now for $t>0$ we have [4] for any solution $x$ of (1.2)

$$
\begin{aligned}
x(t, f, 0)= & {\left[f, G_{0}(t, \cdot), 0\right]=\left[f, G_{0}(t, \cdot r, 1), 0\right] } \\
& +\left[f, G_{0}(t, \cdot r, 2), 0\right] .
\end{aligned}
$$


Since $G(t, s, r, 1)$ satisfies equation (1.2) for all values of $t$ it follows that $\left[f, G_{0}(t, \cdot, r, 1), 0\right]$ has a unique backward extension to $\left[-\Delta_{m}, 0\right]$. Thus it follows from (3.9) and (3.10) that for all $t>0$

$$
x(t, f, 0)=\sum_{j=1}^{n\left(a_{r}\right)} a(f, j) x\left(t, \zeta_{r_{j}}, 0\right)+x_{r}(t) .
$$

From the results of Theorem 2.2 we have that there exists a constant $k(r)$, depending only on $r$, such that for all $t>0$

$$
\left\|x_{r_{t}}\right\| \leqq k(r)\|f\| \exp \left(a_{r} t\right) \text {. }
$$

Summarizing these results we have the following theorem.

THEOREM 3.2. Under the hypothesis of Theorem 2.2 or Theorem 2.3 one has for every $r>H_{2}$ and every solution $x(t, f, 0)$ of $(1.2) a$ unique representation of the form (3.11) where the remainder term $x_{r}$ satisfies the estimate (3.12).

We note finally that the hypothesis of Theorem 2.3 are more restrictive than those of Hale [3] and Cooke [2]. Thus in this case their results are applicable. These results assure us that each of the solutions $x\left(t, \zeta_{r_{j}}, 0\right)$ is asymptotic as $t \rightarrow \infty$ to a solution of (1.1).

\section{REFERENCES}

1. Bellman and Cooke, Differential-Difference Equations, Academic Press, New York, 1963.

2. K. Cooke, Linear functional differential equations of asymptotically autonomous type, J. Differential Equations, 7 (1970), 154-174.

3. J. K. Hale, Linear asymptotically autonomous functional differential equations, Rend. Circ. Mat. Palermo (2), 15 (1966), 331-351.

4. —, Functional Differential Equations, Springer-Verlag, Berlin-HeidelbergNew York, 1971.

5. - Forward and backward continuation for neutral functional differential equations, J. Differential Equations, 7 (1971), 168-181.

6. J. Lillo, Periodic perturbations of $n^{\text {th }}$ order differential difference equations, Amer.

J. Math., XCIV (1972), 651-676.

7. - Almost periodic perturbations of $n^{\text {th }}$ order differential difference equations, Math. Systems Theory, 8 (1974), 207-224.

Received May 28, 1975. This research was supported by National Science Grant GP-28392.

Purdue University 


\title{
PACIFIC JOURNAL OF MATHEMATICS
}

\author{
EDITORS
}

\author{
RICHARD ARENS (Managing Editor) \\ University of California \\ Los Angeles, California 90024
}

\author{
R. A. Beaumont \\ University of Washington \\ Seattle, Washington 98105
}

J. DugundjI

Department of Mathematics

University of Southern California

Los Angeles, California 90007

D. Gilbarg and J. Milgram

Stanford University

Stanford, California 94305

\section{ASSOCIATE EDITORS}
E. F. BECKENBACH
B. H. NEUMANN
F. WOLF
K. Yoshida

\section{SUPPORTING INSTITUTIONS}

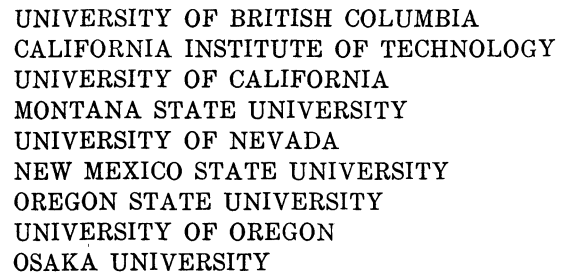

UNIVERSITY OF BRITISH COLUMBIA CALIFORNIA INSTITUTE OF TECHNOLOGY UNIVERSITY OF CALIFORNIA MONTANA STATE UNIVERSITY UNIVERSITY OF NEVADA NEW MEXICO STATE UNIVERSITY OREGON STATE UNIVERSITY UNIVERSITY OF OREGON OSAKA UNIVERSITY

\author{
UNIVERSITY OF SOUTHERN CALIFORNIA \\ STANFORD UNIVERSITY \\ UNIVERSITY OF HAWAII \\ UNIVERSITY OF TOKYO \\ UNIVERSITY OF UTAH \\ WASHINGTON STATE UNIVERSITY \\ UNIVERSITY OF WASHINGTON

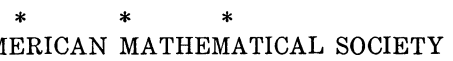

The Supporting Institutions listed above contribute to the cost of publication of this Journal, but they are not owners or publishers and have no responsibility for its content or policies.

Mathematical papers intended for publication in the Pacific Journal of Mathematics should be in typed form or offset-reproduced, (not dittoed), double spaced with large margins. Please do not use built up fractions in the text of your manuscript. You may however, use them in the displayed equations. Underline Greek letters in red, German in green, and script in blue. The first paragraph or two must be capable of being used separately as a synopsis of the entire paper. Items of the bibliography should not be cited there unless absolutely necessary, in which case they must be identified by author and Journal, rather than by item number. Manuscripts, in triplicate, may be sent to any one of the editors. Please classify according to the scheme of Math. Reviews, Index to Vol. 39. All other communications should be addressed to the managing editor, or Elaine Barth, University of California, Los Angeles, California, 90024.

The Pacific Journal of Mathematics expects the author's institution to pay page charges, and reserves the right to delay publication for nonpayment of charges in case of financial emergency.

100 reprints are provided free for each article, only if page charges have been substantially paid. Additional copies may be obtained at cost in multiples of 50 .

The Pacific Journal of Mathematics is issued monthly as of January 1966. Regular subscription rate: $\$ 72.00$ a year (6 Vols., 12 issues). Special rate: $\$ 36.00$ a year to individual members of supporting institutions.

Subscriptions, orders for back numbers, and changes of address should be sent to Pacific Journal of Mathematics, 103 Highland Boulevard, Berkeley, California, 94708.

PUBLISHED BY PACIFIC JOURNAL OF MATHEMATICS, A NON-PROFIT CORPORATION

Printed at Kokusai Bunken Insatsusha (International Academic Printing Co., Ltd.), 8-8, 3-chome, Takadanobaba, Shinjuku-ku, Tokyo 160, Japan.

Copyright (C) 1975 by Pacific Journal of Mathematics Manufactured and first issued in Japan 


\section{Pacific Journal of Mathematics}

\section{Vol. 63, No. 2 \\ April, 1976}

Joseph Anthony Ball and Arthur R. Lubin, On a class of contractive perturbations

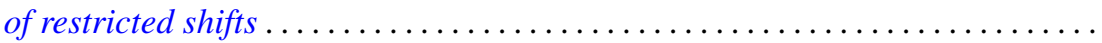

Joseph Becker and William C. Brown, On extending higher derivations generated

by cup products to the integral closure .......................

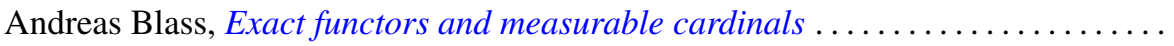

Joseph Eugene Collison, A variance property for arithmetic functions . . . . . . . . . .

Craig McCormack Cordes, Quadratic forms over nonformally real fields with a

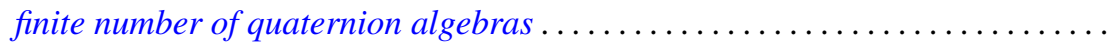

Freddy Delbaen, Weakly compact sets in $H^{1} \ldots \ldots \ldots \ldots \ldots \ldots \ldots \ldots \ldots$

G. D. Dikshit, Absolute Nörlund summability factors for Fourier series ..........

Edward Richard Fadell, Nielsen numbers as a homotopy type invariant. . ........

Josip Globevnik, Analytic extensions of vector-valued functions . . . . . . . . . . . .

Robert Gold, Genera in normal extensions . . . . . . . . . . . . . . . . . . . 389

Solomon Wolf Golomb, Formulas for the next prime

Robert L. Griess, Jr., The splitting of extensions of $S L(3,3)$ by the vector space

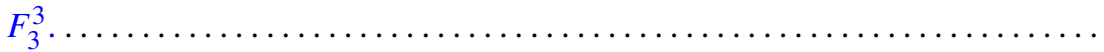

Thomas Alan Keagy, Matrix transformations and absolute summability .........

Kazuo Kishi, Analytic maps of the open unit disk onto a Gleason part.

Kwangil Koh, Jiang Luh and Mohan S. Putcha, On the associativity and commutativity of algebras over commutative rings . ..... . .

James C. Lillo, Asymptotic behavior of solutions of retarded differential difference equations.

John Alan MacBain, Local and global bifurcation from normal eigenvalues ..

Anna Maria Mantero, Sets of uniqueness and multiplicity for $L^{p}$

J. F. McClendon, Embedding metric families

L. Robbiano and Giuseppe Valla, Primary powers of a prime ideal .

Wolfgang Ruess, Generalized inductive limit topologies and barrelledness

properties.

Judith D. Sally, Bounds for numbers of generators of Cohen-Macaulay ideals

Helga Schirmer, Mappings of polyhedra with prescribed fixed points and fixed point indices.

Cho Wei Sit, Quotients of complete multipartite graphs

S. Sznajder and Zbigniew Zielezny, Solvability of convolution equations in $\mathscr{K}_{p}^{\prime}$,

$p>1$.

Mitchell Herbert Taibleson, The existence of natural field structures for finite

dimensional vector spaces over local fields

William Yslas Vélez, A characterization of completely regular fields

P. S. Venkatesan, On right unipotent semigroups ..............

Kenneth S. Williams, A rational octic reciprocity law ............

Robert Ross Wilson, Lattice orderings on the real field .......... 